\title{
EXISTENCE OF SOLUTIONS \\ FOR A FRACTIONAL HYBRID BOUNDARY VALUE PROBLEM VIA MEASURES OF NONCOMPACTNESS IN BANACH ALGEBRAS
}

\author{
Josefa Caballero - Mohamed Abdalla Darwish \\ Kishin SADARANGANI
}

\begin{abstract}
We study the existence of solutions for the following fractional hybrid boundary value problem

$$
\left\{\begin{array}{l}
D_{0^{+}}^{\alpha}\left[\frac{x(t)}{f(t, x(t))}\right]+g(t, x(t))=0, \quad 0<t<1, \\
x(0)=x(1)=0,
\end{array}\right.
$$

where $1<\alpha \leq 2$ and $D_{0^{+}}^{\alpha}$ denotes the Riemann-Liouville fractional derivative. The main tool is our study is the technique of measures of noncompactness in the Banach algebras. Some examples are presented to illustrate our results. Finally, we compare the results of paper with the ones obtained by other authors.
\end{abstract}

\section{Introduction}

Differential equations of fractional order occur more frequently on different research areas and they are very important in the modelling of several physical phenomena [5], [8], [10], [12]. Recently, quadratic perturbations of nonlinear differential equations have attracted much attention to researchers. These types

2010 Mathematics Subject Classification. 45G10, 45M99, 47H09.

Key words and phrases. Banach algebras, Riemann-Liouville fractional derivative, measure of noncompactness, hybrid boundary value problem. 
of equations have been called hybrid differential equations and they have been studied in some papers among them [6], [7], [11], [13], [14].

In [7], Dhage and Lakshmikantham discussed the following first order hybrid differential equation

$$
\left\{\begin{array}{l}
\frac{d}{d t}\left[\frac{x(t)}{f(t, x(t))}\right]=g(t, x(t)), \quad t \in J \\
x\left(t_{0}\right)=x_{0} \in \mathbb{R}
\end{array}\right.
$$

where $J=[0, T), f \in C(J \times \mathbb{R}, \mathbb{R} \backslash\{0\})$ and $g \in C(J \times \mathbb{R}, \mathbb{R})$. The fractional version of the last differential equation, i.e.

$$
\left\{\begin{array}{l}
D_{0^{+}}^{q}\left[\frac{x(t)}{f(t, x(t))}\right]=g(t, x(t)), \quad t \in J \\
x(0)=0
\end{array}\right.
$$

where $0<q<1, f \in C(J \times \mathbb{R}, \mathbb{R} \backslash\{0\})$ and $g \in C(J \times \mathbb{R}, \mathbb{R})$ was studied in [14], where the main ingredient was a fixed point theorem in Banach algebras.

In this paper, we study the existence of solutions for the following fractional hybrid boundary value problem

$$
\left\{\begin{array}{l}
D_{0^{+}}^{\alpha}\left[\frac{x(t)}{f(t, x(t))}\right]+g(t, x(t))=0, \quad 0<t<1, \\
x(0)=x(1)=0
\end{array}\right.
$$

where $1<\alpha \leq 2$ and $D_{0^{+}}^{\alpha}$ is the standard Riemann-Liouville fractional derivative.

Our main tool is a fixed point theorem for the products of two operators which satisfy a condition of Darbo type with respect to a measure of noncompactness.

\section{Basic results}

Firstly, we present some basic facts about fractional calculus theory. This material can be found in [9].

Definition 2.1. The Riemann-Liouville fractional derivative of order $\alpha>0$ of a continuous function $f:(0, \infty) \rightarrow \mathbb{R}$ is defined by

$$
D_{0^{+}}^{\alpha} f(t)=\frac{1}{\Gamma(n-\alpha)}\left(\frac{d}{d t}\right)^{(n)} \int_{0}^{t} \frac{f(s)}{(t-s)^{\alpha-n+1}} d s,
$$

where $n=[\alpha]+1,[\alpha]$ denotes the integer part of $\alpha$, provided that the right side is pointwise defined on $(0, \infty)$.

Definition 2.2. The Riemann-Liouville fractional integral of order $\alpha>0$ of a function $f:(0, \infty) \rightarrow \mathbb{R}$ is defined by

$$
I_{0^{+}}^{\alpha} f(t)=\frac{1}{\Gamma(\alpha)} \int_{0}^{t} \frac{f(s)}{(t-s)^{1-\alpha}} d s
$$


provided that the right side is defined on $(0, \infty)$.

From these definitions, we can obtain the following two lemmas.

Lemma 2.3. Let $\alpha>0$. If $u \in C(0,1) \cap L(0,1)$, then the fractional differential equation

$$
D_{0^{+}}^{\alpha} u(t)=0
$$

has as a unique solutions

$$
u(t)=c_{1} t^{\alpha-1}+c_{2} t^{\alpha-2}+\ldots+c_{n} t^{\alpha-n},
$$

where $c_{i} \in \mathbb{R}, i=1, \ldots, n$ and $n=[\alpha]+1$.

Lemma 2.4. Suppose that $u \in C(0,1) \cap L(0,1)$ with a fractional derivative of order $\alpha>0$ belonging to $C(0,1) \cap L(0,1)$. Then

$$
I_{0^{+}}^{\alpha} D_{0^{+}}^{\alpha} u(t)=u(t)+c_{1} t^{\alpha-1}+c_{2} t^{\alpha-2}+\ldots+c_{n} t^{\alpha-n},
$$

for some $c_{i} \in \mathbb{R}, i=1, \ldots, n$, where $n=[\alpha]+1$.

Now, we present the Green's function of our fractional hybrid boundary value problem. The following lemma appears in [13].

Lemma 2.5. Suppose that $f \in C([0,1] \times \mathbb{R}, \mathbb{R} \backslash\{0\}), y \in C[0,1]$ and $1<\alpha \leq 2$. Then the unique solution of the following boundary value problem

$$
\left\{\begin{array}{l}
D_{0^{+}}^{\alpha}\left[\frac{x(t)}{f(t, x(t))}\right]+y(t)=0, \quad 0<t<1 \\
x(0)=x(1)=0
\end{array}\right.
$$

is

$$
x(t)=f(t, x(t)) \int_{0}^{1} G(t, s) y(s) d s,
$$

where $G(t, s)$ is the Green's function which has the expression

$$
G(t, s)= \begin{cases}\frac{[t(1-s)]^{\alpha-1}-(t-s)^{\alpha-1}}{\Gamma(\alpha)}, & 0 \leq s \leq t \leq 1, \\ \frac{[t(1-s)]^{\alpha-1}}{\Gamma(\alpha)}, & 0 \leq t \leq s \leq 1\end{cases}
$$

The following lemma appears in [2].

Lemma 2.6. The Green function $G(t, s)$ satisfies:

(a) $G \in C([0,1] \times[0,1], \mathbb{R})$,

(b) $G(t, s)>0$ for $t, s \in(0,1)$, and

(c) $\max _{0 \leq t \leq 1} G(t, s)=G(s, s)$, for any $s \in(0,1)$. 
Next, we recollect a few auxiliary facts about measures of noncompactness. Assume that $E$ is a real Banach space with the norm $\|\cdot\|$ and the zero element $\theta$. By $B(x, r)$ we denote the closed ball in $E$ centered at $x$ and with radius $r$. By $B_{r}$ we denote the ball $B(\theta, r)$. If $X$ is a nonempty subset $X$ of $E$ then $\bar{X}$ and Conv $X$ denote the closure and the convex closure of $X$, respectively. By $\operatorname{diam} X$ we will denote the diameter of a bounded set $X$ and $\|\cdot\|$ the norm of $X$, i.e. $\|X\|=\sup \{\|x\|: x \in X\}$. Further, by $\mathfrak{M}_{E}$ we will denote the family of all nonempty and bounded subsets of $E$ and by $\mathfrak{N}_{E}$ its subfamily consisting of all relatively compact subsets.

Throughout this paper, we will accept the following definition of the concept of a measure of noncompactness [3].

Definition 2.7. A mapping $\mu: \mathfrak{M}_{E} \rightarrow \mathbb{R}_{+}=[0, \infty)$ will be called a measure of noncompactness in $E$ if it satisfies the following conditions:

(a) The family ker $\mu$ is nonempty and ker $\mu \subset \mathfrak{N}_{E}$.

(b) $X \subset Y \Rightarrow \mu(X) \leq \mu(Y)$.

(c) $\mu(\bar{X})=\mu(X)$.

(c) $\mu(\operatorname{Conv} X)=\mu(X)$.

(d) $\mu(\lambda X+(1-\lambda) Y) \leq \lambda \mu(X)+(1-\lambda) \mu(Y)$ for $\lambda \in[0,1]$.

(e) If $\left(X_{n}\right)$ is a sequence of closed subsets of $\mathfrak{M}_{E}$ such that $X_{n+1} \subset X_{n}$ and $\lim _{n \rightarrow \infty} \mu\left(X_{n}\right)=0$ then $X_{\infty}=\bigcap_{n=1}^{\infty} X_{n} \neq \phi$.

The family ker $\mu$ appearing in $1^{\circ}$ is called the kernel of the measure of noncompactness $\mu$. Notice that the set $X_{\infty}$ appearing in $6^{\circ}$ belongs to ker $\mu$. Indeed, since $\mu\left(X_{\infty}\right) \leq \mu\left(X_{n}\right)$ for any $n=1,2, \ldots$, we infer that $\mu\left(X_{\infty}\right)=0$ and this means that $X_{\infty} \in \operatorname{ker} \mu$.

In what follows, we will assume that the space $E$ has structure of Banach algebra. In such a case, we denote by $x y$ the products of two elements $x, y \in E$ and by $X Y$ the product of two subsets $X$ and $Y$ of $E$, i.e. $X Y=\{x y: x \in X$, $y \in Y\}$.

Now, we recall the following concept appearing in [4] which will play an important role in our considerations.

Definition 2.8. We say that a measure of noncompactness $\mu$ defined on the Banach algebra $E$ satisfies condition $(m)$ if, for any $X, Y \in \mathfrak{M}_{E}$, the following inequality is satisfied

$$
\mu(X Y) \leq\|X\| \mu(Y)+\|Y\| \mu(X)
$$

In [1], the authors proved the following generalization of Darbo's fixed point theorem. 
TheOREm 2.9 [1, Theorem 2.2]). Let $\Omega$ be a nonempty, bounded, closed and convex subset of a Banach space $E$ and let $F: \Omega \rightarrow \Omega$ be a continuous operator satisfying

$$
\mu(F X) \leq \varphi(\mu(X)),
$$

for any nonempty subset $X$ of $\Omega$, where $\mu$ is an arbitrary measure of noncompactness and $\varphi: \mathbb{R}_{+} \rightarrow \mathbb{R}_{+}$is a nondecreasing function such that $\lim _{n \rightarrow \infty} \varphi^{n}(t)=0$ for each $t \in \mathbb{R}_{+}$, where $\varphi^{n}$ denotes the $n$-iteration of $\varphi$. Then $T$ has at least one fixed point in $\Omega$.

Moreover, in [1] the authors proved the following lemma which will be useful in our considerations.

Lemma 2.10 ([1, Lemma 2.1]). Let $\varphi: \mathbb{R}_{+} \rightarrow \mathbb{R}_{+}$be a nondecreasing and upper semicontinuous function. Then the following conditions are equivalent:

(a) $\lim _{n \rightarrow \infty} \varphi^{n}(t)=0$, for any $t \geq 0$,

(b) $\varphi(t)<t$, for any $t>0$.

In this paper, we will work in the space $C[0,1]$ consisting of all real functions defined and continuous on the interval $[0,1]$ with the usual supremum norm

$$
\|x\|=\sup \{|x(t)|: t \in[0,1]\},
$$

for $x \in C[0,1]$. Notice that the space $C[0,1]$ has also structure of Banach algebra, where the multiplication is defined as the usual product of real functions.

Next, we will present the measure of noncompactness in $C[0,1]$ which will be used in our study. Let us fix a set $X \in \mathfrak{M}_{C[0,1]}$. For $x \in X$ and $\varepsilon>0$, we denote by $\omega(x, \varepsilon)$ the modulus of continuity of $x$, i.e.

$$
\omega(x, \varepsilon)=\sup \{|x(t)-x(s)|: t, s \in[0,1],|t-s| \leq \varepsilon\} .
$$

Further, put

$$
\omega(X, \varepsilon)=\sup \{\omega(x, \varepsilon): x \in X\} \quad \text { and } \quad \omega_{0}(X)=\lim _{\varepsilon \rightarrow 0} \omega(X, \varepsilon) .
$$

In [3], it is proved that $\omega_{0}(X)$ is a measure of noncompactness in $C[0,1]$.

THeOREM 2.11. The measure of noncompactness $\omega_{0}$ on $C[0,1]$ satisfies condition $(m)$.

Proof. Fix $X, Y \in \mathfrak{M}_{C[0,1]}, \varepsilon>0$ and $t, s \in[0,1]$ with $|t-s| \leq \varepsilon$. Then, for $x \in X$ and $y \in Y$, we have

$$
\begin{aligned}
|x(t) y(t)-x(s) y(s)| & \leq|x(t) y(t)-x(t) y(s)|+|x(t) y(s)-x(s) y(s)| \\
& =|x(t)||y(t)-y(s)|+|y(s)||x(t)-x(s)| \\
& \leq\|x\| \omega(y, \varepsilon)+\|y\| \omega(x, \varepsilon) .
\end{aligned}
$$


From this, it follows that

$$
\omega(x y, \varepsilon) \leq\|x\| \omega(y, \varepsilon)+\|y\| \omega(x, \varepsilon)
$$

and, consequently,

$$
\omega(X Y, \varepsilon) \leq\|X\| \omega(Y, \varepsilon)+\|Y\| \omega(X, \varepsilon) .
$$

Taking $\varepsilon \rightarrow 0$ in the last inequality, we get

$$
\omega_{0}(X Y) \leq\|X\| \omega_{0}(Y)+\|Y\| \omega_{0}(X)
$$

This completes the proof.

\section{Main results}

By commodity, we will denote by $\mathcal{A}$ the following class of functions which will be used later,

$$
\mathcal{A}=\left\{\varphi: \mathbb{R}_{+} \rightarrow \mathbb{R}_{+}: \varphi \text { is nondecreasing and } \lim _{n \rightarrow \infty} \varphi^{n}(t)=0 \text { for ny } t \in \mathbb{R}_{+}\right\}
$$

Remark 3.1. Notice that if $\lambda \in[0,1]$ and $\varphi \in \mathcal{A}$ then $\lambda \varphi \in \mathcal{A}$. In fact, it is clear that $\lambda \varphi$ is nondecreasing. Moreover, $(\lambda \varphi)(t) \leq \varphi(t)$ for any $t \in \mathbb{R}_{+}$. By using mathematical induction, we can prove that $(\lambda \varphi)^{(n)}(t) \leq \lambda \varphi^{(n)}(t)$ and this proves that $\lim _{n \rightarrow \infty}(\lambda \varphi)^{n}(t)=0$ for any $t \in \mathbb{R}_{+}$. Therefore, $\lambda \varphi \in \mathcal{A}$.

Now, we are ready to prove the existence result of equation (1.1). We consider the following assumptions:

$\left(\mathrm{a}_{1}\right) f \in C([0,1] \times \mathbb{R}, \mathbb{R} \backslash\{0\})$ and $g \in C([0,1] \times \mathbb{R}, \mathbb{R})$.

$\left(\mathrm{a}_{2}\right)$ There exist nonnegative constants $k_{1}$ and $k_{2}$ such that $|f(t, 0)| \leq k_{1}$ and $|g(t, 0)| \leq k_{2}$ for any $t \in[0,1]$.

$\left(\mathrm{a}_{3}\right)$ The functions $f$ and $g$ satisfy:

$$
\begin{aligned}
\left|f\left(t, x_{1}\right)-f\left(t, x_{2}\right)\right| & \leq \varphi_{1}\left(\left|x_{1}-x_{2}\right|\right) \\
\left|g\left(t, x_{1}\right)-g\left(t, x_{2}\right)\right| & \leq \varphi_{2}\left(\left|x_{1}-x_{2}\right|\right)
\end{aligned}
$$

respectively, for any $t \in[0, T]$ and $x_{1}, x_{2} \in \mathbb{R}$, where $\varphi_{1}, \varphi_{2} \in \mathcal{A}$ and $\varphi_{1}$ is continuous.

$\left(\mathrm{a}_{4}\right)$ There exists $r_{0}>0$ satisfying the inequality

$$
\left(\varphi_{1}(r)+k_{1}\right) \cdot\left(\varphi_{2}(r)+k_{2}\right) \leq \frac{\Gamma(2 \alpha)}{\Gamma(\alpha)} r
$$

and, moreover

$$
\left(\varphi_{2}\left(r_{0}\right)+k_{2}\right) \leq \frac{\Gamma(\alpha)}{\Gamma(2 \alpha)} .
$$


THEOREM 3.2. Under assumptions $\left(\mathrm{a}_{1}\right)-\left(\mathrm{a}_{4}\right)$, equation (1.1) has at least one solution in $C[0,1]$.

Proof. In virtue of Lemma 2.5, any solution of equation (1.1) must satisfy the following integral equation

$$
x(t)=f(t, x(t)) \int_{0}^{1} G(t, s) g(s, x(s)) d s,
$$

where $G(t, s)$ is the Green's function appearing in Section 2. Therefore, the solutions of (1.1) are the fixed points of the operator defined on $C[0,1]$ by the formula

$$
(\mathcal{T} x)(t)=f(t, x(t)) \int_{0}^{1} G(t, s) g(s, x(s)) d s .
$$

Consider the operators $\mathcal{F}$ and $\mathcal{G}$ defined on $C[0,1]$ by $(\mathcal{F} x)(t)=f(t, x(t))$ and $(\mathcal{G} x)(t)=\int_{0}^{1} G(t, s) g(s, x(s)) d s$, for any $x \in C[0,1]$ and $t \in[0,1]$. Then, $\mathcal{T} x=$ $(\mathcal{F} x) \cdot(\mathcal{G} x)$ for any $x \in C[0,1]$.

By $\left(\mathrm{a}_{1}\right)$ and Lemma 2.6, it is easy to see that the operators $\mathcal{F}$ and $\mathcal{G}$ apply $C[0,1]$ into itself and, since the product of continuous functions is a continuous function, $\mathcal{T}$ applies $C[0,1]$ into itself.

Next, we fix $x \in C[0,1]$ and $t \in[0,1]$. Then, taking into account our assumptions, we get

$$
\begin{aligned}
|(\mathcal{T} x)(t)|= & |(\mathcal{F} x)(t)||(\mathcal{G} x)(t)| \\
= & |f(t, x(t))|\left|\int_{0}^{1} G(t, s) g(s, x(s)) d s\right| \\
\leq & {[|f(t, x(t))-f(t, 0)|+|f(t, 0)|] } \\
& \cdot\left|\int_{0}^{1} G(t, s)(g(s, x(s))-g(s, 0)) d s+\int_{0}^{1} G(t, s) g(s, 0) d s\right| .
\end{aligned}
$$

Since $G(t, s) \geq 0$ for $t, s \in[0,1]$ (see Lemma 2.6), we have

$$
\begin{aligned}
|(\mathcal{T} x)(t)| \leq & \left(\phi_{1}(|x(t)|)+|f(t, 0)|\right) \\
& \cdot\left[\int_{0}^{1} G(t, s)|g(s, x(s))-g(s, 0)| d s+\int_{0}^{1} G(t, s)|g(s, 0)| d s\right] \\
\leq & \left(\phi_{1}(|x(t)|)+|f(t, 0)|\right)\left[\int_{0}^{1} G(t, s) \varphi_{2}(|x(s)|) d s+k_{2} \int_{0}^{1} G(t, s) d s\right] \\
\leq & \left(\phi_{1}(\|x\|)+k_{1}\right)\left(\phi_{2}(\|x\|)+k_{2}\right) \int_{0}^{1} G(t, s) d s .
\end{aligned}
$$

Since $\max _{0 \leq t \leq 1} G(t, s)=G(s, s)$, for any $s \in(0,1)$, we have

$$
\|\mathcal{T} x\| \leq\left(\phi_{1}(\|x\|)+k_{1}\right)\left(\phi_{2}(\|x\|)+k_{2}\right) \int_{0}^{1} G(s, s) d s .
$$


Taking into account that

$$
\int_{0}^{1} G(s, s) d s=\frac{1}{\Gamma(\alpha)} \int_{0}^{1} s^{\alpha-1}(1-s)^{\alpha-1} d s=\frac{1}{\Gamma(\alpha)} B(\alpha, \alpha)=\frac{\Gamma(\alpha)}{\Gamma(2 \alpha)},
$$

from inequality (3.2), it follows that

$$
\|\mathcal{T} x\| \leq \frac{\Gamma(\alpha)}{\Gamma(2 \alpha)}\left(\phi_{1}(\|x\|)+k_{1}\right)\left(\phi_{2}(\|x\|)+k_{2}\right) .
$$

From assumption $\left(a_{4}\right)$, we infer that the operator $\mathcal{T}$ transforms $B_{r_{0}}$ into itself. Moreover, from the last estimates, we get

$$
\left\|\mathcal{F} B_{r_{0}}\right\| \leq\left(\phi_{1}\left(r_{0}\right)+k_{1}\right) \quad \text { and } \quad\left\|\mathcal{G} B_{r_{0}}\right\| \leq \frac{\Gamma(\alpha)}{\Gamma(2 \alpha)}\left(\phi_{2}\left(r_{0}\right)+k_{2}\right) .
$$

In the sequel, we will prove that the operator $\mathcal{F}$ is continuous on the ball $B_{r_{0}}$. To do this, we fix $\varepsilon>0$ and we take $x, y \in B_{r_{0}}$ with $\|x-y\| \leq \varepsilon$. Then, for $t \in[0,1]$, we have

$$
\begin{aligned}
|(\mathcal{F} x)(t)-(\mathcal{F} y)(t)| & =|f(t, x(t))-f(t, y(t))| \\
& \leq \varphi_{1}(|x(t)-y(t)|) \leq \varphi_{1}(\|x-y\|) \leq \varphi_{1}(\varepsilon) .
\end{aligned}
$$

Since $\varphi_{1} \in \mathcal{A}$ it is easy to see that $\varphi_{1}(t)<t$ for any $t>0$ (because, in contrary case, we find $t_{0}>0$ such that $\varphi_{1}\left(t_{0}\right)<t_{0}$. Since $\varphi_{1}$ is nondecreasing, $\left(\varphi_{1}^{n}\left(t_{0}\right)\right)$ is a nondecreasing sequence satisfying $t_{0} \leq \varphi_{1}^{n}\left(t_{0}\right)$ for any $n \in \mathbb{N}$, and, consequently, $0<t_{0} \leq \lim _{t \rightarrow \infty} \varphi_{1}^{n}\left(t_{0}\right)$ and this contradicts the fact that $\left.\varphi_{1} \in \mathcal{A}\right)$. From $\varphi_{1}(t)<t$ for any $t>0$, we deduce that $\varphi_{1}(0)=0$ and $\lim _{t \rightarrow 0} \varphi_{1}(t)=0$. Therefore, $\varphi_{1}$ is continuous at $t=0$ and, from the last inequality, we infer that $\|\mathcal{F} x-\mathcal{F} y\| \rightarrow 0$ when $\varepsilon \rightarrow 0$.

Notice that in this argument we do not used the assumption about the continuity of $\varphi_{1}$ (assumption $\left(a_{3}\right)$ ). This proves that $\mathcal{F}$ is continuous on $B_{r_{0}}$.

In order to prove that $\mathcal{G}$ is continuous on $B_{r_{0}}$, we fix $\varepsilon>0$ and for $x, y \in B_{r_{0}}$ with $\|x-y\| \leq \varepsilon$, and $t \in[0,1]$, we have

$$
\begin{aligned}
|(\mathcal{G} x)(t)-(\mathcal{G} y)(t)| & =\left|\int_{0}^{1} G(t, s) g(s, x(s)) d s-\int_{0}^{1} G(t, s) g(s, y(s)) d s\right| \\
& \leq \int_{0}^{1} G(t, s)|g(s, x(s))-g(s, y(s))| d s \\
& \leq \omega_{g}(\varepsilon) \int_{0}^{1} G(t, s) d s
\end{aligned}
$$

where $\left.\omega_{g}(\varepsilon)=\sup \left\{|g(s, x)-g(s, y)|: s \in[0,1], x, y \in\left[-r_{0}, r_{0}\right],|x-y| \leq \varepsilon\right)\right\}$.

Since $g(t, x)$ is uniformly continuous on bounded subsets of $[0,1] \times \mathbb{R}$, we infer that $\omega_{g}(\varepsilon) \rightarrow 0$ as $\varepsilon \rightarrow 0$, and, from the last inequality, it follows that $\|\mathcal{G} x-\mathcal{G} y\| \rightarrow 0$ as $\varepsilon \rightarrow 0$. This proves that $\mathcal{G}$ is continuous on $B_{r_{0}}$. Therefore, $\mathcal{T}=\mathcal{F} \cdot \mathcal{G}$ is continuous on $B_{r_{0}}$. 
Next, we will estimate the quantity related to the modulus of continuity for the operators $\mathcal{F}$ and $\mathcal{G}$ on $B_{r_{0}}$. To do this, we fix a nonempty subset $X$ of $B_{r_{0}}$ and $\varepsilon>0$. Then, for $x \in X$ and $t_{1}, t_{2} \in[0,1]$ with $\left|t_{1}-t_{2}\right| \leq \varepsilon$, we get

$$
\begin{aligned}
\left|(\mathcal{F} x)\left(t_{1}\right)-(\mathcal{F} x)\left(t_{2}\right)\right| & =\left|f\left(t_{1}, x\left(t_{1}\right)\right)-f\left(t_{2}, x\left(t_{2}\right)\right)\right| \\
& \leq\left|f\left(t_{1}, x\left(t_{1}\right)\right)-f\left(t_{2}, x\left(t_{1}\right)\right)\right|+\left|f\left(t_{2}, x\left(t_{1}\right)\right)-f\left(t_{2}, x\left(t_{2}\right)\right)\right| \\
& \leq \omega(f, \varepsilon)+\varphi_{1}\left(\left|x\left(t_{1}\right)-x\left(t_{2}\right)\right|\right) \leq \omega(f, \varepsilon)+\varphi_{1}(\omega(X, \varepsilon)),
\end{aligned}
$$

where $\omega(f, \varepsilon)=\sup \left\{|f(r, x)-f(s, x)|: r, s \in[0,1],|r-s| \leq \varepsilon, x \in\left[-r_{0}, r_{0}\right]\right\}$. This means that

$$
\omega(\mathcal{F} x, \varepsilon) \leq \omega(f, \varepsilon)+\varphi_{1}(\omega(X, \varepsilon)) .
$$

From this, it follows that

$$
\omega(\mathcal{F} X, \varepsilon) \leq \omega(f, \varepsilon)+\varphi_{1}(\omega(X, \varepsilon)) .
$$

Since $f(t, x)$ is uniformly continuous on bounded subsets of $[0,1] \times \mathbb{R}$, we deduce that $\omega(f, \varepsilon) \rightarrow 0$ as $\varepsilon \rightarrow 0$, and, from the last inequality, since $\varphi_{1}$ is continuous, we obtain

$$
\omega_{0}(\mathcal{F} X) \leq \varphi_{1}\left(\lim _{\varepsilon \rightarrow 0} \omega(X, \varepsilon)\right)=\varphi_{1}\left(\omega_{0}(X)\right)
$$

Now, we estimate the quantity $\omega_{0}$ for the operator $\mathcal{G}$. To do this, we fix a nonempty subset $X$ of $B_{r_{0}}$ and $\varepsilon>0$. Then, for $x \in X$ and $t_{1}, t_{2} \in[0,1]$ with $\left|t_{1}-t_{2}\right| \leq \varepsilon$, we have

$$
\begin{aligned}
\left|(\mathcal{G} x)\left(t_{1}\right)-(\mathcal{G} x)\left(t_{2}\right)\right| & =\left|\int_{0}^{1} G\left(t_{1}, s\right) g(s, x(s)) d s-\int_{0}^{1} G\left(t_{2}, s\right) g(s, x(s)) d s\right| \\
& \leq \int_{0}^{1}\left|G\left(t_{1}, s\right)-G\left(t_{2}, s\right)\right||g(s, x(s))| d s .
\end{aligned}
$$

Put $L=\sup \left\{|g(t, x)|: t \in[0,1], x \in\left[-r_{0}, r_{0}\right]\right\}$. Since $g$ is continuous on the compact set $[0,1] \times\left[-r_{0}, r_{0}\right], L<\infty$. Then, from the last estimate, we have

$\left|(\mathcal{G} x)\left(t_{1}\right)-(\mathcal{G} x)\left(t_{2}\right)\right| \leq L \int_{0}^{1}\left|G\left(t_{1}, s\right)-G\left(t_{2}, s\right)\right| d s \leq L \int_{0}^{1} \omega(G, \varepsilon) d s \leq L \omega(G, \varepsilon)$, where $\omega(G, \varepsilon)=\sup \left\{\left|G\left(\tau_{1}, s\right)-G\left(\tau_{2}, s\right)\right|: \tau_{1}, \tau_{2}, s \in[0,1],\left|\tau_{1}-\tau_{2}\right| \leq \varepsilon\right\}$. This gives us that

$$
\omega(\mathcal{G} x, \varepsilon) \leq L \omega(G, \varepsilon)
$$

and, consequently,

$$
\omega(\mathcal{G} X, \varepsilon) \leq L \omega(G, \varepsilon) .
$$

Since $G(t, s)$ is uniformly continuous on $[0,1] \times[0,1]$, we infer that $\omega(G, \varepsilon) \rightarrow 0$ as $\varepsilon \rightarrow 0$, and, the last inequality gives us that

$$
\omega_{0}(\mathcal{G} X)=0
$$


Finally, we will estimate the quantity $\omega_{0}$ for the operator $\mathcal{T}$ on $B_{r_{0}}$. Let $X$ be a nonempty subset of $B_{r_{0}}$. Taking into account Theorem 2.11, we have

$$
\begin{aligned}
\omega_{0}(\mathcal{T} X) & =\omega_{0}(\mathcal{F} X \cdot \mathcal{G} X) \\
& \leq\|\mathcal{F} X\| \omega_{0}(\mathcal{G} X)+\|\mathcal{G} X\| \omega_{0}(\mathcal{F} X) \leq\left\|\mathcal{F} B_{r_{0}}\right\| \omega_{0}(\mathcal{G} X)+\left\|\mathcal{G} B_{r_{0}}\right\| \omega_{0}(\mathcal{F} X) .
\end{aligned}
$$

By (3.3)-(3.5), we infer that

$$
\omega_{0}(\mathcal{T} X) \leq \frac{\Gamma(\alpha)}{\Gamma(2 \alpha)}\left(\varphi_{2}\left(r_{0}\right)+k_{2}\right) \varphi_{1}\left(\omega_{0}(X)\right)
$$

By assumptions $\left(\mathrm{a}_{4}\right), \frac{\Gamma(\alpha)}{\Gamma(2 \alpha)}\left(\varphi_{2}\left(r_{0}\right)+k_{2}\right) \leq 1$ and, taking into account Remark 3.1, $\frac{\Gamma(\alpha)}{\Gamma(2 \alpha)}\left(\varphi_{2}\left(r_{0}\right)+k_{2}\right) \varphi_{1} \in \mathcal{A}$. Finally, by using Theorem 2.9 , the operator $\mathcal{T}$ has at least one fixed point in $B_{r_{0}}$.

\section{Example}

Before to present an example illustrating our results, we need some properties about the inverse tangent function.

Definition 4.1. A function $f: \mathbb{R}_{+} \rightarrow \mathbb{R}_{+}$is said to be subadditive if

$$
f(x+y) \leq f(x)+f(y), \quad \text { for any } x, y \in \mathbb{R}_{+} .
$$

Lemma 4.2. Suppose that $f: \mathbb{R}_{+} \rightarrow \mathbb{R}_{+}$is subadditive and $y \leq x$ then

$$
f(x)-f(y) \leq f(x-y) .
$$

Proof. In fact, since $f(x)=f(x-y+y) \leq f(x-y)+f(y)$ it follows the desired result.

REMARK 4.3. Notice that from Lemma 4.2, we infer that if $f: \mathbb{R}_{+} \rightarrow \mathbb{R}_{+}$is subadditive then

$$
|f(x)-f(y)| \leq f(|x-y|), \quad \text { for any } x, y \in \mathbb{R}_{+} .
$$

Lemma 4.4. Let $f: \mathbb{R}_{+} \rightarrow \mathbb{R}_{+}$be a concave function with $f(0)=0$. Then $f$ is subadditive.

Proof. We take $x, y \in \mathbb{R}_{+}$. The concavity of $f$ and the fact that $f(0)=0$ give us

$f(x)=f\left(\frac{x}{x+y}(x+y)+\frac{y}{x+y} \cdot 0\right) \geq \frac{x}{x+y} f(x+y)+\frac{y}{x+y} f(0)=\frac{x}{x+y} f(x+y)$

and

$f(y)=f\left(\frac{x}{x+y} \cdot 0+\frac{y}{x+y}(x+y)\right) \geq \frac{x}{x+y} f(0)+\frac{y}{x+y} f(x+y)=\frac{y}{x+y} f(x+y)$. 
Adding these inequalities, we have

$$
f(x)+f(y) \geq \frac{x}{x+y} f(x+y)+\frac{y}{x+y} f(x+y)=f(x+y) .
$$

This completes the proof.

REMARK 4.5. Since the function $\varphi: \mathbb{R}_{+} \rightarrow[0, \pi / 2)$ defined by $\varphi(t)=\arctan t$ is concave (because $\varphi^{\prime \prime}=-2 t /\left(\left(1+t^{2}\right)^{2}\right) \leq 0$ for $\left.t \in \mathbb{R}_{+}\right)$and $\varphi(0)=0$, Lemma 4.4 says us that $\varphi$ is subadditive. By using Remark 4.3, we have

$$
|\arctan t-\arctan s| \leq \arctan (|t-s|), \quad \text { for any } t, s \in \mathbb{R}_{+} .
$$

Since $\arctan t<t$ for any $t>0$ (because if $h(t)=t-\arctan t$ for $t \geq 0$, then $h^{\prime}(t)=1-1 /\left(1+t^{2}\right)>0$ for $t>0$ and, therefore, $h(0)<h(t)$ for $t>0$. Consequently, arctan $t<t$ for $t>0)$ and $\varphi(t)=\arctan t$ for $t \in \mathbb{R}_{+}$is continuous, Lemma 2.10 gives us $\lim _{n \rightarrow \infty} \varphi^{n}(t)=0$ for any $t \in \mathbb{R}_{+}$. Moreover, it is clear that $\varphi(t)=\arctan t$ is nondecreasing and, therefore, $\varphi \in \mathcal{A}$.

Now, we are ready to present an example where our results can be applied.

EXAMPLE 4.6. Consider the following fractional hybrid boundary value problem

$$
\left\{\begin{array}{l}
D_{0^{+}}^{3 / 2}\left[\frac{x(t)}{1 / 2+\arctan |x(t)|}\right]+\arctan |x(t)|=0, \quad 0<t<1, \\
x(0)=x(1)=0 .
\end{array}\right.
$$

Notice that, (4.1) is a particular case of (1.1), where $\alpha=3 / 2, f(t, x)=1 / 2+$ $\arctan |x|$ and $g(t, x)=\arctan |x|$.

It is clear that $f \in C([0,1] \times \mathbb{R}, \mathbb{R} \backslash\{0\})$ and $g \in C([0,1] \times \mathbb{R}, \mathbb{R})$ and, moreover, $|f(t, 0)|=1 / 2=k_{1}$ and $|g(t, 0)|=0=k_{2}$. Thus, assumptions $\left(\mathrm{a}_{1}\right)$ and $\left(\mathrm{a}_{2}\right)$ of Theorem 3.2 are satisfied.

On the other hand, taking into account Remark 4.5, for any $x_{1}, x_{2} \in \mathbb{R}$ and $t \in \mathbb{R}_{+}$, we have

$$
\begin{aligned}
\left|f\left(t, x_{1}\right)-f\left(t, x_{2}\right)\right| & =|\arctan | x_{1}|-\arctan | x_{2}|| \\
& \leq \arctan \left(|| x_{1}|-| x_{2}||\right) \leq \arctan \left(\left|x_{1}-x_{2}\right|\right),
\end{aligned}
$$

where we have used the nondecreasing character of the inverse tangent function and the fact that ||$x_{1}|-| x_{2}|| \leq\left|x_{1}-x_{2}\right|$. Therefore, $\varphi_{1}(t)=\arctan t$ and $\varphi_{1} \in \mathcal{A}$ (see Remark 4.5). Moreover, it is clear that $\varphi_{1}$ is continuous.

Further, notice that, for any $x_{1}, x_{2} \in \mathbb{R}$ and $t \in \mathbb{R}_{+}$, we have

$$
\begin{aligned}
\left|g\left(t, x_{1}\right)-g\left(t, x_{2}\right)\right| & =|\arctan | x_{1}|-\arctan | x_{2}|| \\
& \leq \arctan \left(|| x_{1}|-| x_{2}||\right) \leq \arctan \left(\left|x_{1}-x_{2}\right|\right)
\end{aligned}
$$

and, in this case, $\varphi_{2}(t)=\arctan t$ with $\varphi_{2} \in \mathcal{A}$. This proves that assumption $\left(\mathrm{a}_{3}\right)$ of Theorem 3.2 is satisfied. 
In our case, the inequality appearing in assumption $\left(\mathrm{a}_{4}\right)$ of Theorem 3.2 has the expression

$$
\left(\arctan r+\frac{1}{2}\right)(\arctan r) \leq \frac{\Gamma(3)}{\Gamma(3 / 2)} r .
$$

It is easy to see that this inequality is satisfied for $r_{0}=1 / \sqrt{3}$ and, moreover,

$$
\arctan r_{0}=\arctan \frac{1}{\sqrt{3}}=0.523598 \leq \frac{\Gamma(3)}{\Gamma(3 / 2)}=\frac{2}{0.88623}=2.256750 .
$$

This proves that assumption $\left(\mathrm{a}_{4}\right)$ of Theorem 3.2 is satisfied.

Therefore, Theorem 3.2 says us that (4.1) has at least one solution $x \in C[0,1]$ such that $\|x\| \leq 1 / \sqrt{3}$.

\section{Final remarks}

In [13], the authors studied equation (1.1) under other assumptions. More precisely, they proved the following result.

THEOREM 5.1 ([13, Theorem 3.1]). Suppose that the following assumptions are satisfied:

$\left(\mathrm{H}_{1}\right) f \in C([0,1] \times \mathbb{R}, \mathbb{R} \backslash\{0\})$ and $g \in C([0,1] \times \mathbb{R}, \mathbb{R})$.

$\left(\mathrm{H}_{2}\right)$ There exists a constant $L>0$ such that

$$
|f(t, x)-f(t, y)| \leq L|x-y|
$$

for any $t \in[0,1]$ and $x, y \in \mathbb{R}$.

$\left(\mathrm{H}_{3}\right)$ There exists a function $h \in L^{1}\left([0,1], \mathbb{R}_{+}\right)$such that

$$
|g(t, x)| \leq h(t), \quad \text { for any } t \in[0,1] .
$$

$\left(\mathrm{H}_{4}\right) L T\|h\|_{1}<1$, where $T=\int_{0}^{1} G(s, s) d s$.

Then equation $(1.1)$ has a solution in $C([0,1], \mathbb{R})$.

Now, we present an example which can be treated by Theorem 3.2 and it cannot studied by using Theorem 5.1. Previously, by using similar arguments to the ones used for the inverse tangent function can be proved that the functions $\varphi_{1}(t)=\tanh t=\left(e^{2 t}-1\right) /\left(e^{2 t}+1\right)$ and $\varphi_{2}(t)=\ln (1+t)$ belong to the class $\mathcal{A}$.

EXAMPLE 5.2. Consider the following fractional hybrid boundary value problem:

$$
\left\{\begin{array}{l}
D_{0^{+}}^{3 / 2}\left[\frac{x(t)}{1 / 4+\tanh |x(t)|}\right]+\ln (1+|x(t)|)=0, \quad 0<t<1, \\
x(0)=x(1)=0 .
\end{array}\right.
$$

Notice that, equation (5.1) is a particular case of (1.1), where $\alpha=3 / 2, f(t, x)=$ $1 / 4+\tanh |x|$ and $g(t, x)=\ln (1+|x|)$. 
It is clear that assumption $\left(\mathrm{a}_{1}\right)$ of Theorem 3.2 is satisfied, and, moreover, $|f(t, 0)|=1 / 4=k_{1}$ and $|g(t, 0)|=0=k_{2}$. Since the function $\varphi_{1}(t)=\tanh t$ is concave and $\varphi_{1}(0)=0$, by Lemma $4.4, \varphi_{1}$ is subadditive and, therefore, for any $x_{1}, x_{2} \in \mathbb{R}$ and $t \in \mathbb{R}_{+}$, we have

$$
\left|f\left(t, x_{1}\right)-f\left(t, x_{2}\right)\right|=\leq \tanh \left(|| x_{1}|-| x_{2}||\right) \leq \tanh \left(\left|x_{1}-x_{2}\right|\right),
$$

and $\varphi_{1}(r)=\tanh r$. Notice that $\varphi_{1} \in \mathcal{A}$ and $\varphi_{1}$ is continuous.

On the other hand, for any $x_{1}, x_{2} \in \mathbb{R}$ and $t \in \mathbb{R}_{+}$, we have

$$
\left|g\left(t, x_{1}\right)-g\left(t, x_{2}\right)\right|=\left|\ln \left(1+\left|x_{1}\right|\right)-\ln \left(1+\left|x_{2}\right|\right)\right| .
$$

Suppose that $\left|x_{1}\right|>\left|x_{2}\right|$ (same argument works for $\left|x_{2}\right|>\left|x_{1}\right|$ ), then

$$
\begin{aligned}
\left|g\left(t, x_{1}\right)-g\left(t, x_{2}\right)\right| & =\left|\ln \left(\frac{1+\left|x_{1}\right|}{1+\left|x_{2}\right|}\right)\right| \\
& =\ln \left(\frac{1+\left|x_{2}\right|}{1+\left|x_{2}\right|}+\frac{\left|x_{1}\right|-\left|x_{2}\right|}{1+\left|x_{2}\right|}\right)=\ln \left(1+\frac{\left|x_{1}\right|-\left|x_{2}\right|}{1+\left|x_{2}\right|}\right) \\
& \leq \ln \left(1+\left(\left|x_{1}\right|-\left|x_{2}\right|\right)\right) \leq \ln \left(1+\left|x_{1}-x_{2}\right|\right),
\end{aligned}
$$

where we have used the nondecreasing character of $\varphi_{2}(t)=\ln (1+t)$ for $t \in \mathbb{R}_{+}$ and the fact that $\left|x_{1}\right|-\left|x_{2}\right| \leq\left|x_{1}-x_{2}\right|$. In our case, $\varphi_{2}(t)=\ln (1+t)$ for $t \in \mathbb{R}_{+}$ and it is easy to see that $\varphi_{2} \in \mathcal{A}$.

The inequality appearing in assumption $\left(a_{4}\right)$ of Theorem 3.2 has the form

$$
\left(\tanh r+\frac{1}{4}\right)(\ln (1+r)) \leq \frac{\Gamma(3)}{\Gamma(3 / 2)} r .
$$

It is easy to see that this inequality is satisfied by $r_{0}=1$ and, moreover,

$$
\ln \left(1+r_{0}\right) \frac{\Gamma(\alpha)}{\Gamma(2 \alpha)}=\ln (2) \cdot \frac{\Gamma(3 / 2)}{\Gamma(3)}=\ln (2) \cdot 0.443115 \leq 1 .
$$

Therefore, by Theorem 3.2, (5.1) has at least one solution $x \in C([0,1], \mathbb{R})$ with $\|x\| \leq 1$.

Notice that in our case the function $g(t, x)=\ln (1+|x|)$ does not satisfy assumption $\left(\mathrm{H}_{4}\right)$ of Theorem 5.1 and, therefore, equation (5.1) cannot be treated by the results of [13].

\section{REFERENCES}

[1] A. Aghajani, J. Banaś And N. Sebzali, Some generalizations of Darbo fixed point theorem and applications, Bull. Belg. Math. Soc. Simon Stevin 20 (2013), 345-358.

[2] Z. BAi AND H. Lü, Positive solutions for boundary value problem of nonlinear fractional differential equation, J. Math. Anal. Appl. 311 (2005), 495-505.

[3] J. BAnaś And K. Goebel, Measures of Noncompactness in Banach Spaces, Lecture Notes in Pure and Applied Mathematics, vol. 60, Marcel Dekker, New York, 1980. 
[4] J. BANAŚ AND L. Olszowy, On a class of measures of noncompactness in Banach algebras and their application to nonlinear integral equations, Z. Anal. Anwend. 28 (2009), 475-498.

[5] K. Diethelm And A.D. FReed, On the solutions of nonlinear fractional-order differential equations used in the modelling of viscoplasticity, Scientific Computing in Chemical Engineering II (1999), 217-224.

[6] B.C. Dhage, A nonlinear alternative in Banach algebras with applications to functional differential equations, Nonlinear Funct. Anal. Appl. 8 (2004), 563-575.

[7] B.C. Dhage And V. Lakshmikantham, Basic results on hybrid differential equations, Nonlinear Anal. Hybrid Syst. 4 (2010), 414-424.

[8] W.G. Glöckle and T.F. Nonnenmacher, A fractional calculus approach to selfsimilar protein dynamics, Biophys J. 68 (1995), 46-53.

[9] A.A. Kilbas, H.M. Srivastava and J.J. Trujillo, Theory and Applications of Fractional Differential Equations, North-Holland Mathematics Studies, vol. 204, Elsevier Science B.V., Amsterdam, 2006.

[10] F. MAINARDi, Fractional calculus: some basic problems in continuum and statistical mechanics, Fractals and Fractional Calculus in Continuum Mechanics (Udine, 1996), CISM Courses and Lectures, vol. 378, Springer, Vienna, 1997, pp. 291-348.

[11] H. Noroozi, A. Ansari And M.S. Dahaghin, Existence results for the distributed order fractional hybrid differential equations, Art. ID 163648, Abstr. Appl. Anal. (2012), $16 \mathrm{pp}$.

[12] R. Metzler, W. Schick, H.G. Kilian and T.F. Nonnenmacher, Relaxation in filled polymers: A fractional calculus approach, J. Chem. Phys. 103 (1995), 7180-7186.

[13] S. Sun, Y. ZhaO, Z. HAN AND Y. Li, The existence of solutions for boundary value problem of fractional hybrid differential equations, Commun. Nonlinear Sci. Numer. Simul. 17 (2012), 4961-4967.

[14] Y. Zhao, S. Sun, Z. Han ANd Q. Li, Theory of fractional hybrid differential equations, Comput. Math. Appl. 62 (2011), 1312-1324.

Mohamed Abdalla Darwish

Department of Mathematics

Sciences Faculty for Girls

King Abdulaziz University

Jeddah, SAUDI ARABIA

and

Department of Mathematics

Faculty of Science

Damanhour University

Damanhour, EGYPT

E-mail address: dr.madarwish@gmail.com

Josefa Caballero and Kishin Sadarangani

Departamento de Matemáticas

Universidad de Las Palmas de Gran Canaria

Campus de Tafira

Baja 35017 Las Palmas

de Gran Canaria, SPAIN

E-mail address: fefi@dma.ulpgc.es, ksadaran@dma.ulpgc.es

TMNA : Volume $43-2014-\mathrm{N}^{\circ} 2$ 